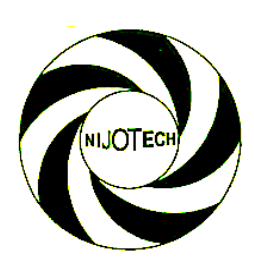

Nigerian Journal of Technology (NIJOTECH)

Vol. 38, No. 2, April 2019, pp. $\mathbf{4 6 5}$ - $\mathbf{4 6 9}$

Copyright@ Faculty of Engineering, University of Nigeria, Nsukka

Print ISSN: 0331-8443, Electronic ISSN: 2467-8821 www.nijotech.com

http://dx.doi.org/10.4314/njt.v38i2.24

\title{
A COMPARATIVE STUDY OF TIME SERIES ANALYSIS FOR FORECASTING ENERGY DEMAND IN NIGERIA
}

\author{
I. K. Okakwu1,*, E. S. Oluwasogo ${ }^{2}$, A. E. Ibhaze ${ }^{3}$ and A. L. Imoize ${ }^{4}$ \\ 1, Dept. of Electrical and Electronic Engineering, Univ. of Benin, Benin City, Edo State, Nigeria \\ 2, Dept. of Electrical and Computer Engr, KWara State Univ., Malete, KWara State, NIGERIA \\ 3,4, DEPT. OF ELECTRICAL AND ELECTRONICS ENGR, UNIV. OF LAGOS, AKOKA, LAGOS, LAGOS STATE, NIGERIA. \\ E-mail addresses: ${ }^{1}$ igokakwu@yahoo.com, ${ }^{2}$ emmanueloluwasogu@yahoo.com, 3 eibhaze@unilag.edu.ng, \\ 4 aimoize@unilag.edu.ng
}

\begin{abstract}
The complexity of the Nigerian Power Sector due to industry deregulation and abrupt variations in electricity demand and the ever increasing population density requires urgent research attention and industry action. Getting an accurate model to fit the energy demand pattern has become imperative and the need for an appropriate model cannot be overemphasized. This article therefore presents a comparative study on time series modelling of average and peak load forecasting in Nigeria. Data from the National Control Center (NCC), Oshogbo was harvested and analyzed using Harvey, Autoregressive, Moving Average and Exponential Smoothing Time Series Models while using R-Statistics for model simulation. The comparative performance showed that the Harvey Model best predicted load demand with least values of 111.0188 Root Mean Squared Error, (RMSE), 9.1095 Mean Absolute Error (MAE), 2.3579 Mean Absolute Percentage Error (MAPE) and 0.0156 Theil Inequality Coefficient (TIC) for average load and 117.4345 RMSE, 10.183 MAE, 17.01 Mean Absolute Percentage Error (MAPE), and 0.015 TIC for peak load.
\end{abstract}

Keywords: Forecasting, Electric load demand, Harvey model, Autoregressive model, Moving Average model, Time Series model, Exponential Smoothing model, Theil Inequality Coefficient.

\section{INTRODUCTION}

A key index used in the determination of a Nations wellbeing in terms of development is its quest for energy demand. The global trend of technology evolution, technology transfer, integration and utilization places huge demand on energy availability. The per capita index and other economic metrics are therefore dependent on the reliability of the electrical power sector of any nation as everything seem to rise and fall on the degree of its efficiency. Electric power supply is therefore rated as one of the most essential commodities for any nation's development [1]. A quick overview on nation building informs that efficient power supply results in quality health care and economic growth to mention a few [2]. Development results in increase in power demand, which invariably requires planning ahead of time to meet the present and future demand of uninterruptible power supply
[3]. Therefore, forecasting of electric energy demand is an important process in the planning of electricity utility companies [4]. In addition, accurate forecast leads to increase reliability of power supply, correct decision making for future development, quality savings in operation and maintenance costs [5]. As electric power demand increases, energy consumption assessment becomes imperative which could be carried out either hourly, daily, weekly, monthly or yearly [6]. Accurate load forecasting is vital because an overestimation will lead to economic waste since huge investment is needed for the development of excessive power infrastructure, while underestimating may result in the degeneration and possible collapse of the existing infrastructure as underinvestment may ensue thereby leading to load shedding or consumer disconnection [7]. The categorization of load forecasting is usually based on usage measured in

${ }^{*}$ Corresponding author, tel: +234 - $805-664-7215$ 
short, medium and long term. Short term load forecasting is usually deployed when scheduling the generation and transmission of electricity. Medium term load forecasting is employed when scheduling fuel purchase while long term forecasting is used to model power supply and power delivery [8].

Research community is saturated with diverse approach to investigating the best estimate for load forecast for which time series analysis has been adopted as the most prominent. Idoniboyeobu et al. [9], carried out a study on long term electric load forecasting on the Nigerian power system using the modified form of exponential regression model. The model was used to predict residential, commercial and industrial load demand. In [10], Garch model was used to estimate the volatility of the marketplace, while Harvey logistic model was used to forecast electricity demand and supply in Nigeria between 2005 and 2026. Residential electricity utilization in Nigeria was modelled and predicted using multiple/quadratic regression models in [11]. Results showed that the quadratic regression model outperformed multiple regression model. In [12], electricity demand forecast in Tamale Ghana using ARIMA model was studied. The study employed secondary data spanning from 1990 to 2013 , and the result showed that both domestic and commercial demand were increasing more rapidly than industrial sector demand. [13] carried out a comparative study on medium term load forecasting using Artificial Neural Network, ANN and regression model. Results showed that ANN-model was more superior to the regression model for load forecasting. Predicting electricity consumption using regression, Kalman filter adaptation algorithm and ANN was investigated in [14]. The report indicated that Kalman filter adaptation algorithm was best performing in terms of future prediction of electricity consumption. The aim of this paper is to carry out comparative prediction for load demand using the Moving Average (MA) model, Autoregressive Model (AM), Exponential Smoothing Model and the Harvey Model (HM). Load forecasting model accuracy will be evaluated using statistical tools for which the best model would be recommended for use in load the forecasting of the Nigerian Power system

\section{METHODOLOGY}

\subsection{Data Collection}

The data used in this study comprises of average and peak load demand from 2008 to 2017. They were obtained from the National Control Centre, Oshogbo, Nigeria as shown in Table 1.

Table 1: Average and Peak load demand in Nigeria (MW)

\begin{tabular}{ccc}
\hline Year & Average load & Peak load \\
\hline 1998 & 2357 & 2448 \\
1999 & 2300 & 2458 \\
2000 & 2161 & 2499 \\
2001 & 2449 & 2934 \\
2002 & 3019 & 3223 \\
2003 & 3200 & 3479 \\
2004 & 3316 & 3428 \\
2005 & 3420 & 3775 \\
2006 & 3267 & 3682 \\
2007 & 3267 & 3600 \\
2008 & 3267 & 3682 \\
2009 & 3393 & 3600 \\
2010 & 3607 & 3804 \\
2011 & 3800 & 4089 \\
2012 & 3765 & 4054 \\
2013 & 4064 & 4458 \\
2014 & 4171 & 4487 \\
2015 & 4444 & 4811 \\
2016 & 4166 & 5075 \\
2017 & 4641 & 5222 \\
\hline
\end{tabular}

\subsection{The Exponential Smoothing Model}

The exponential smoothing model is given as in equation (1).

$$
\widehat{Y}_{t+1}=\alpha Y_{t}+(1-\alpha) \hat{Y}_{t}
$$

where $\hat{Y}_{t+1}=$ forecast value at time $t$ over the period $\mathrm{t}+1 ; Y_{t}=$ actual value at $\mathrm{t} ; \alpha=$ smoothing constant $(0<a<1)$.

Substitution into previous forecasting value, results in (2).

$$
\begin{aligned}
\hat{Y}_{t+1}=\alpha \cdot Y_{t}+\alpha \cdot & (1-\alpha) \cdot \hat{Y}_{t}+\alpha \cdot(1-\alpha)^{2} \cdot \hat{Y}_{t-1} \\
& +\cdots \alpha \cdot(1-\alpha)^{t-1} \cdot Y_{1} \\
& +(1-\alpha)^{t} \cdot Y_{0}
\end{aligned}
$$

In compact form, equation (2) is reduced to the form of (3).

$$
\hat{Y}_{t+1}=\alpha \sum_{k=0}^{t-1}(1-\alpha)^{k} Y_{t-k}+(1-\alpha)^{t} Y_{0}
$$

\subsection{Moving Average Model}

The moving average is deduced by compacting the mean value for a specific set of variables and then adopting for forecasting the next period. The governing equation is presented in (4)-(6).

$$
\begin{gathered}
\hat{Y}_{t+1}=\frac{Y_{t}+Y_{t-1}+\cdots+Y_{t-n+1}}{n} \\
\hat{Y}_{t}=\frac{Y_{t-1}+Y_{t-2}+\cdots+Y_{t-n}}{n}
\end{gathered}
$$




$$
\hat{Y}_{t+1}=\hat{Y}_{t}+\frac{Y_{t}-Y_{t-n}}{n}
$$

where $\hat{Y}_{t+1}=$ forecast value at $\mathrm{t}+1 ; Y_{t}=$ actual value at $\mathrm{t}$ and $\mathrm{n}=$ degree or measure of observations

\subsection{Autoregressive Model}

The first order autoregressive model is given by (7)

$$
Y_{t}=\beta_{o}+\beta_{1} Y_{t-1}+e_{i}
$$

The estimated model is given in (8) and subsequently in (9). Substituting (8) into (9) gives (10).

$$
\hat{Y}_{t}=\hat{\beta}_{o}+\hat{\beta}_{1} Y_{t-1}
$$

where

$$
\begin{gathered}
\hat{\beta}_{o}=\bar{Y}_{t}-\hat{\beta}_{1} \bar{Y}_{t-1} \\
\hat{\beta}_{1}=\frac{n \sum_{t=2}^{n} Y_{t} Y_{t-1}-\left(\sum_{t=2}^{n} Y_{t}\right)\left(\sum_{t=2}^{n} Y_{t-1}\right)}{n \sum_{t=2}^{n} Y_{t-1}^{2}-\left(\sum_{t=2}^{n} Y_{t-1}\right)^{2}}
\end{gathered}
$$

\subsection{Harvey Model}

The Harvey Model is given by (11).

$$
\ln y_{t}=a \ln Y_{t-1}+b+c t+\varepsilon
$$

and $Y_{t}=Y_{t}-Y_{t-1}$

Let $P_{i}=\ln \left(\frac{Y_{t}}{Y_{t-1}}\right)$ and $q_{i}=\ln \left(Y_{t-1}\right)$ then equation becomes (12).

$$
P_{i}=a q_{i}+b+c t+\varepsilon
$$

The error is, given in (13), and by a suitable assumption, (15) is derived from (14)

$$
\sum_{i=1}^{n} \varepsilon_{i}^{2}=\sum_{i=1}^{n}\left(P_{i}-a q_{i}-b-c t\right)^{2}
$$

Let

$$
\begin{gathered}
u=\sum_{i=1}^{n} \varepsilon^{2} \\
\frac{\partial u}{\partial a}=-2\left[\sum_{i=1}^{n} P_{i} q_{i}-a \sum_{i=1}^{n} q_{i}-b \sum_{i=1}^{n} q_{i}-c \sum_{i=1}^{n} t_{i}\right] \\
\frac{\partial u}{\partial b}=-2\left[\sum_{i=1}^{n} P_{i} q_{i}-a \sum_{i=1}^{n} q_{i}-n b-c \sum_{i=1}^{n} t_{i}\right] \\
\frac{\partial u}{\partial c}=-2\left[\sum_{i=1}^{n} P_{i} t_{i}-a \sum_{i=1}^{n} q_{i} t_{i}-b \sum_{i=1}^{n} t_{i}-c \sum_{i=1}^{n} t_{i}^{2}\right]
\end{gathered}
$$

if $\frac{\partial u}{\partial a}=\frac{\partial u}{\partial b}=\frac{\partial u}{\partial c}=0$ and rearranged in matrix form in (15).

$$
\left[\begin{array}{l}
a \\
b \\
c
\end{array}\right]=\left[\begin{array}{ccc}
\sum_{i=1}^{n} q_{i}^{2} & \sum_{i=1}^{n} q_{i} & \sum_{i=1}^{n} q_{i} t_{i} \\
\sum_{i=1}^{n} q_{i} & n & \sum_{i=1}^{n} t_{i} \\
\sum_{i=1}^{n} q_{i} t_{i} & \sum_{i=1}^{n} t_{i} & \sum_{i=1}^{n} t_{i}^{2}
\end{array}\right]^{-1}\left[\begin{array}{c}
\sum_{i=1}^{n} P_{i} q_{i} \\
\sum_{i=1}^{n} P_{i} \\
\sum_{i=1}^{n} P_{i} t_{i}
\end{array}\right]
$$

The solution of equation (15) gives the constants of the model.

\subsection{Performance Evaluation of Model}

The statistical tools used to estimate the model's performance are herein represented taking note that the smaller the error, the better the forecasting model. Root Mean Square Error (RMSE). The statistical tools are given in equations (19) to (22).

$$
R M S E=\sqrt{\frac{\sum_{i=1}^{n}\left(\hat{y}_{t}-y_{t}\right)^{2}}{n}}
$$

Mean Absolute Error (MAE)

$$
M A E=\sum_{t=1}^{n} \frac{\left|\hat{y}_{t}-y_{t}\right|}{n}
$$

Mean Absolute Percentage Error (MAPE)

$$
\text { MAPE }=\sum_{t=1}^{n} \frac{\left|\frac{\hat{y}_{t}-y_{t}}{y_{t}}\right|}{n} \times 100 \%
$$

Theil Inequality Coefficient (TIC)

$$
\text { TIC }=\frac{\sum_{t=1}^{n} \sqrt{\frac{\left(\hat{y}_{t}-y_{t}\right)^{2}}{n}}}{\sqrt{\frac{\sum_{t=1}^{n} \hat{y}_{t}^{2}}{n}}+\sqrt{\frac{\sum_{t=1}^{n} y_{t}^{2}}{n}}}
$$

where $\hat{y}_{t}=$ actual value $y_{t}=$ forecasted value $n=$ number of observations $t=$ period

\section{RESULT AND DISCUSSION}

The R-Statistics was used to simulate the average and peak load data using Harvey model, Autoregression model, Moving average model and Exponential Smoothing model. The model performance is achieved by computing the corresponding RMSE, MAE, MAPE and TIC, respectively.

Figure 1 shows comparison of the average load (actual load) and forecasted values for the models from 1998 to 2017, while Table-2 gives the model performance for average load respectively.

Fig 1 shows the result of actual average load and estimated average load by the respective models. From Table-2, the Harvey model gave the least values for all the performance evaluation of the models, followed by AR, Exponential Smoothing and MA models respectively.

Table 2: Model performance for Average Load

\begin{tabular}{ccccc}
\hline MODEL & MAPE & MAE & RMSE & TIC \\
\hline HARVEY & 2.3579 & 9.1095 & 111.0188 & 0.0156 \\
AR & 3.8113 & 11.6899 & 206.5682 & 0.029 \\
MA & 7.2494 & 12.7379 & 297.3273 & 0.0405 \\
EXP. S & 6.2481 & 14.8771 & 283.9837 & 0.0399
\end{tabular}

$\mathrm{AR}=$ Auto Regression; $\mathrm{MA}=$ Moving Average... 


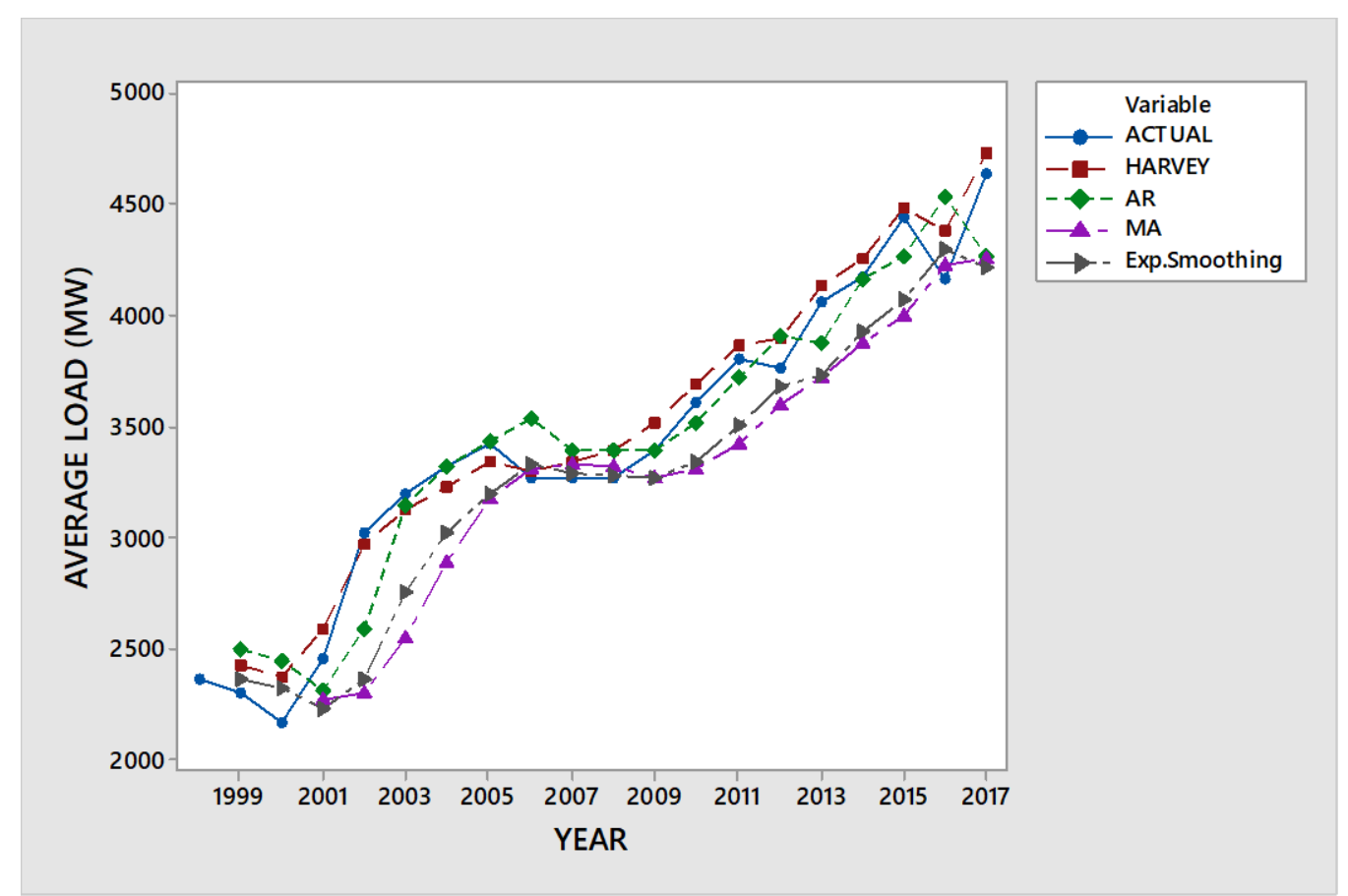

Figure 1: Actual and Predicted Average Load in Nigeria from 1998-2017

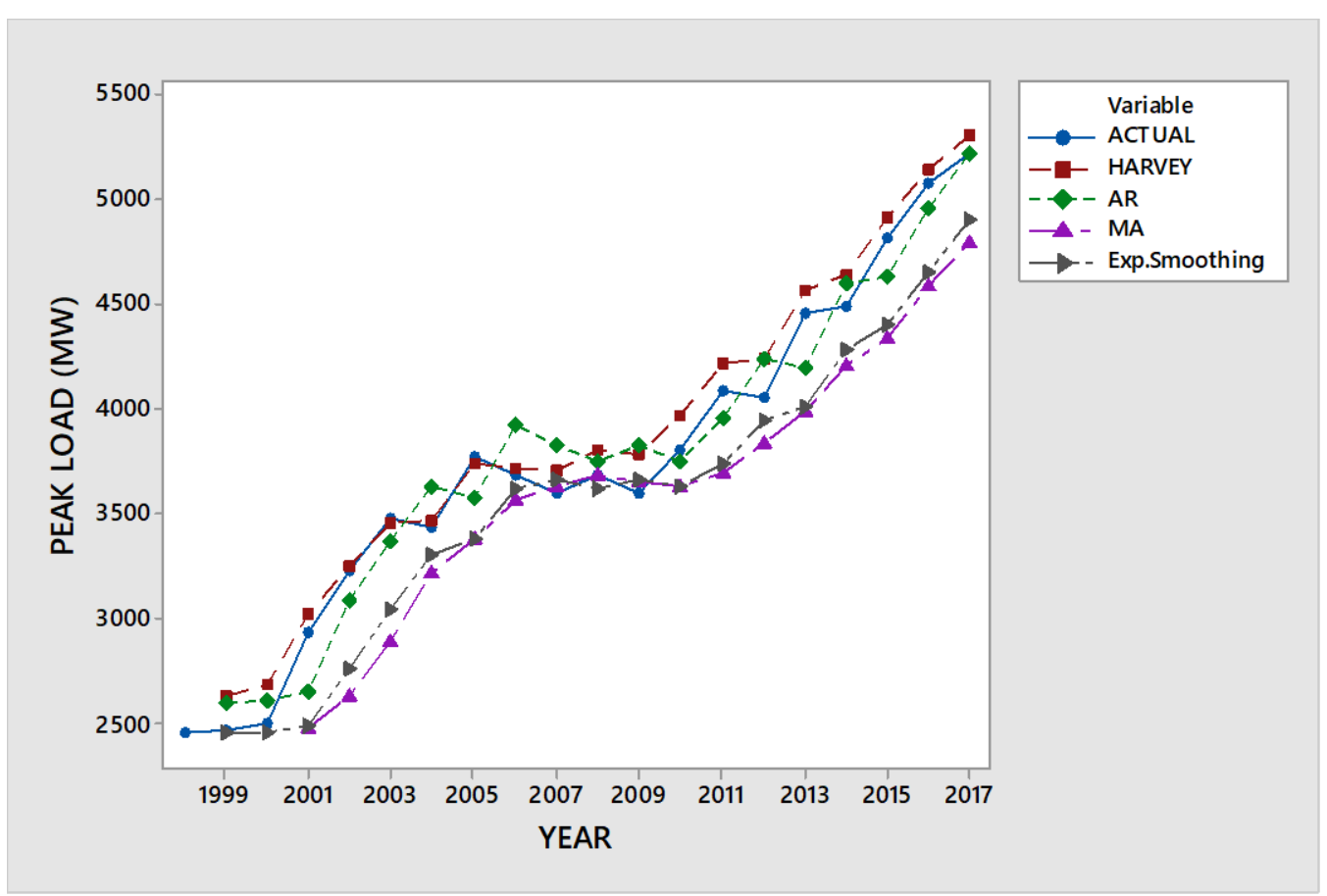

Figure 2: Actual and Predicted Peak Load in Nigeria from 1998-2017

Table 3: Model performance for Peak Load

\begin{tabular}{ccccc}
\hline MODEL & MAPE & MAE & RMSE & TIC \\
\hline HARVEY & 17.01 & 10.183 & 117.4345 & 0.015 \\
AR & 20.91 & 12.08305 & 173.4002 & 0.0221 \\
MA & 28.55 & 12.7379 & 371.2269 & 0.0457 \\
EXP. S & 23.09 & 15.6253 & 297.2053 & 0.0378 \\
\hline
\end{tabular}

AR=Auto Regression; MA=Moving Average...
Also, Fig 2 depicts the result of actual peak load and predicted peak load by the different models. Table-2 also shows that Harvey model has the least values for all the performance evaluation of the models, followed by $A R$, Exponential Smoothing and MA models respectively.

As shown in the Tables 2 and 3, the Harvey model gave the best result among the four contending 
models with the least MAPE value of 2.3579, MAE value of 9.1095, RMSE value of 111.0188 and TIC value of 0.0156 for average load, while for peak load, the values are MAPE (17.01), MAE (10.183), RMSE (117.4345) and TIC (0.015), respectively. Hence, on the basis of the performance validation for the different models, the Harvey model was seen as the best model to predict both average and peak load demand.

\section{CONCLUSION}

Electric load forecasting is essential for planning in advance to meet the continuous supply of electricity demand. This article has articulated the validation of time series modelling for average and peak load demand forecasting in Nigeria. The time series model used include Harvey model, Autoregressive model, Moving Average model and Exponential Smoothing model. The data used was obtained from the National Control Centre (NCC), Oshogbo, Nigeria between 1998-2017. The result obtained shows that Harvey model has a better prediction accuracy when compared with other models. Future works could focus on the optimization of the Harvey model for improved prediction accuracy.

\section{REFERENCES}

[1] P. A. Owusu and S. A. Sarkodie, "A review of renewable energy sources, sustainability issues and climate change mitigation", cogent Engineering, 3: 1-14, 2016.

[2] O. C. Nwankwo and B. O. Njogo, "The effect of electricity supply on industrial production within the Nigerian economy (1970-2010), Journal of Energy Technologies and Policy, 3: 34-42, 2013.

[3] Y. A. Adeshina and I. O. Osunmakinde, "Shortterm multiple forecasting of electric energy loads for sustainable demand planning in smart grids for smart homes", Journal of Sustainability, 9: 1-27, 2017.

[4] T. A. Briggs and K. Ugorji, 2017, "Assessment of electricity demand and prediction model for the future: Rivers state", European Journal of Mechanical Engineering Research, 4: 1-23, 2017
[5] E. Almeshaiei and H. Soltan, "A methodology for electric power load forecasting", Alexandria Engineering Journal, 50: 137-144, 2011.

[6] M. U. Fahad and N. Arbab,"Factors affecting short term load forecasting", Journal of Clean Energy Technologies, 2: 305-309., 2014.

[7] N. L. Rajakovic and V. M. Shiljkut, "Long-term forecasting of annual peak load considering effects of demand-side programs", Journal of Modern Power Systems and Clean Energy, 6: 145-157, 2018.

[8] V. Gupta and S. Pal, "An Overview of Different Types of Load Forecasting Methods and the Factors Affecting the Load Forecasting", International Journal for Research in Applied Science \& Engineering Technology (IJRASET), 5: 729-733, 2017.

[9] D. C. Idoniboyeobu, A. J. Ogunsakin and B. A. Wokoma, "Forecasting of electricity energy demand in Nigeria using modified form of exponential model", American Journal of Engineering Research, 7: 122-135, 2018.

[10] B. O. Oyelami and A. A. Adewunmi, "Models for forecasting the demand and supply of electricity in Nigeria", American Journal of modelling and optimization, 2: 25-33, 2014.

[11] I. A. Ezenugu, S. C. Nwokonkwo and I. Markson, "Modelling and Forecasting of residential electricity consumption in Nigeria using Multiple and Quadratic regression models". American Journal of Software Engineering and Applications, 6: 99-104, 2017.

[12] S. Katara, A. Faisal and G. M. Engmann, "A Time Series Analysis of electricity demand in Tamale, Ghana", International Journal of Statistics and Applications, 4: 269-275, 2014.

[13] A. S. Isaac, E. Adetiba, I. A. Odigwe and F. C. Felly-Njoku, "A Comparative study of regression analysis and artificial neural network methods for medium-term load forecasting", Indian Journal of Science and Technology: 10: 1-7, 2017.

[14] P. Ozoh, S. Abd-Rahman and J. Labadin, "Predicting electricity consumption: A comparative analysis of the accuracy of various computational technique", $g^{\text {th }}$ International Conference on IT in Asia, Kuching, Malaysia. 2015. 